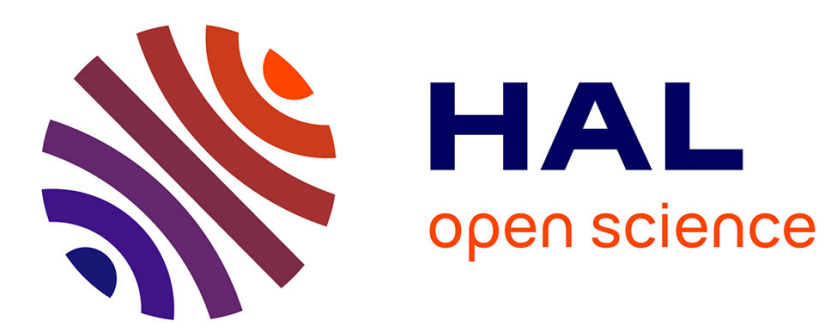

\title{
Karl Polanyi et la pédagogie de l'incohérence
}

Bernard Gazier, Marguerite Mendell

\section{To cite this version:}

Bernard Gazier, Marguerite Mendell. Karl Polanyi et la pédagogie de l'incohérence. Annals of Public and Cooperative Economics, 2009, 80 (1), pp.1-35. hal-00635090

\section{HAL Id: hal-00635090 https://hal.science/hal-00635090}

Submitted on 26 Oct 2011

HAL is a multi-disciplinary open access archive for the deposit and dissemination of scientific research documents, whether they are published or not. The documents may come from teaching and research institutions in France or abroad, or from public or private research centers.
L'archive ouverte pluridisciplinaire HAL, est destinée au dépôt et à la diffusion de documents scientifiques de niveau recherche, publiés ou non, émanant des établissements d'enseignement et de recherche français ou étrangers, des laboratoires publics ou privés. 
Article pour Annals of Public and Cooperative Economics/Annales de l'économie publique, sociale et coopérative

\title{
Karl Polanyi et la pédagogie de l'incohérence
}

\author{
B. GAZIER
}

M. MENDELL

Version révisée, juillet 2008

\section{Résumé}

Cet article vise à examiner de manière critique et constructive l'actualité de la pensée de Karl Polanyi pour comprendre le développement de l'économie plurielle au XXIe siècle et pour agir sur celui-ci. Il montre la très grande actualité de cette pensée, ainsi que la nécessité de s'intéresser à l'œuvre entière de Karl Polanyi, et pas seulement à "La grande transformation ». Partant des usages multiples du concept d'encastrement et de désencastrement, il relie les propositions inspirées par Karl Polanyi sur "le processus institué de démocratisation économique »à des développements récents tant pratiques que théoriques : les «marchés transitionnels du travail » et la «nouvelle finance publique ».

\section{Introduction}

Une série de travaux récents ont permis de souligner l'actualité de Karl Polanyi (en français, voir par exemple la Revue du MAUSS 2007, R. Sobel (dir) 2007, Maucourant 2005, ainsi que l'introduction et la postface de Polanyi 2008 ; en anglais, Bugra et Agartan (dir) 2007, et Harvey, Ramlogan et Randles (dir) 2007). Ecrits dans le contexte du renouveau de la socio économie institutionnaliste, ils ont été l'occasion de faire le point sur ses apports comme sur les zones d'ombre, d'indécision ou d'évolution de sa pensée. L'objet de cet article est de revenir, non pas tant sur son diagnostic célèbre faisant apparaître la marchandisation du monde et les réactions qu'elle suscite comme problème central de notre époque, que sur les perspectives d'action qu'il a pu contribuer à dégager et leur rapport à l'économie sociale.

Pour avancer dans cette tâche, deux principes apparaissent utiles. Tout d'abord, ils est possible de mettre à profit de recul dont nous disposons désormais, et de relier les fils de l'œuvre, au-delà des thèses célèbres de La grande transformation, afin de prendre en compte aussi bien le Polanyi soucieux des «conditions de vie de l'homme » que le réformateur social 
engagé dans des projets pédagogiques (Mendell 1994). On peut alors mettre en évidence la portée programmatique de son propos, et notamment montrer qu'il s'agit d'une pensée de l'incohérence, qu'il s'agisse de la dénoncer ou au contraire de prendre appui sur elle.

Ensuite, on peut réinsérer ces apports dans une série de dynamiques conceptuelles qu'ils ont impulsées ou infléchies jusqu'à nos jours. Un tel travail conceptuel, identifiant des transmissions et des connexions, peut aussi bien s'appuyer sur des auteurs qui se réfèrent explicitement à Polanyi, mais n'appartiennent pas à sa mouvance, que sur des auteurs dont le projet est parallèle voire convergent mais qui explorent des champs différents ou se réfèrent à d'autres corpus théoriques.

Cette contribution adopte cette double perspective en se focalisant sur les usages opérationnels du concept d'encastrement et de désencastrement. Ce concept, central dans l'œuvre de Polanyi et dans la socio - économie institutionnaliste, a reçu récemment une attention soutenue et a fait l'objet de clarifications importantes (Caillé et Laville 2007, Le Velly 2007). Prenant appui sur elles, cet article interroge plus systématiquement le rôle de l'Etat et du non-marchand dans l'économie plurielle d'aujourd'hui et dans la promotion d'une «démocratisation instituée ». (Mendell 2007 a et c) En un premier temps, on mettra en évidence la richesse actuelle mais aussi les complexités et parfois les incertitudes du concept d'encastrement / désencastrement pour les relier à la dynamique de l'œuvre de Polanyi ; en un second temps, on identifiera et on discutera quatre perspectives de réencastrement. On partira de stratégies de promotion de l'économie sociale ${ }^{1}$ dans sa spécificité régionale et nationale, avec l'exemple de l'expérience québécoise où elle prend une plus grande envergure aujourd'hui, à la fois comme acteur socio-économique, et comme partie prenante d'une reconfiguration des relations entre l'Etat, le marché et la société civile. Ensuite on s'intéressera à deux stratégies transversales, l'une portant sur le marché du travail: les «marchés transitionnels du travail» et l'autre sur les fondements de l'action collective : les «biens publics globaux », pour aboutir à la dimension proprement politique, débouchant sur le socialisme associationniste esquissé par Karl Polanyi.

\section{Le concept d'encastrement au tournant du millénaire : l'actualité renforcée et paradoxale de Karl Polanyi}

Il n'est pas question dans le cadre de cet article de reprendre en détail les travaux très nombreux qui d'une manière ou d'une autre ont discuté, précisé ou critiqué le concept d'encastrement / désencastrement. Après avoir récapitulé une série de clarifications qui constituent désormais un acquis, nous souhaitons mettre en évidence les difficultés et les conditions de sa mobilisation opérationnelle, tout d'abord en montrant l'usage qu'a pu en faire G. Esping - Andersen dans son effort pour penser la variété des régimes d'Etat providence dans les pays de l'OCDE, et ensuite en le reliant au paradigme de l'économie

\footnotetext{
${ }^{1}$ Nous adopterons tout au long de cet article une définition extensive de l'économie sociale, incluant les associations, les coopératives et les mutuelles, et visant à dépasser les catégorisations juridico organisationnelles pour en souligner la visée transformatrice de l'ordre institutionnel existant. Sur la signification de cette vision extensive, qui nous semble fidèle aux orientations de K. Polanyi, voir Eme et Laville 2006, et la synthèse de Laville, Lévesque et Mendell 2007.
} 
plurielle. Relativement récentes et très synthétiques, les formulations de B. Lévesque dans cette même revue (Lévesque 2003) nous serviront ici de relais. Il sera alors possible de mettre en évidence la spécificité de la représentation polanyienne du changement institutionnel, pour montrer comment les perspectives d'action qu'il ouvre font de l'incohérence à la fois un objet de dénonciation et un levier.

\section{I.1. Le concept d'encastrement, quelques clarifications.}

Dans la lignée des reformulations et au-delà des critiques présentées par Mark Granovetter (Granovetter 1985 et 2000), il est désormais acquis que le contenu du concept d' « embeddedness » est double (LeVelly 2007 ; Caillé et Laville 2007). L'idée générale de l'encastrement est que les activités économiques ${ }^{2}$ ne peuvent être pensées isolément, au rebours des présentations traditionnelles des sciences économiques. Elles sont « enchâssées », «scellées» dans les institutions et des rapports sociaux extra -économiques qui les contraignent à des degrés divers mais aussi les rendent viables. Ce second rôle n'a été mis en avant que récemment. Il convient ainsi de distinguer l' «encastrement - étayage » de l' « encastrement - insertion », pour reprendre le vocabulaire de R. Le Velly (op. cit.). Le premier est l' « indispensable référence au contexte » social et institutionnel (ibid p. 190), et désigne l'intégration «des actions économiques dans des réseaux de relations sociales interpersonnelles qu'il convient de cerner à partir de leur structure » (Caillé et Laville op. cit. p.97). Le monde social est ici en quelque sorte le pré - requis cognitif et relationnel qui rend possibles les relations économiques, si calculatrices et individualistes soient-elles. Mais c'est la dimension de la contrainte qui domine dans les conceptions originelles de K. Polanyi (Polanyi 1983 (1944)). On peut alors parler d' «encastrement - insertion », désignant «l'inscription de l'économie dans des règles sociales, culturelles et politiques qui régissent certaines formes de production et de circulation des biens et des services » (ibid). K. Polanyi livre alors son diagnostic célèbre sur notre époque, qui serait celle des tendances généralisées $\mathrm{au}$ "désencastrement », autrement dit à l'émancipation de la sphère marchande. Traitant de la terre, de la monnaie et des travailleurs comme des choses, faisant apparaître les interdépendances des marchés et le rôle exclusif du gouvernement par les prix, l'utopie du «marché autorégulateur» vient en quelque sorte inverser les dépendances et les encadrements : c'est alors la société qui serait encastrée dans le marché (Polanyi 1983, op. cit., p. 88). La perspective devient alors doublement dramatique. D'abord parce que cette autonomisation vient menacer les conditions de vie des groupes dépourvus de pouvoir vis-àvis du marché ; ensuite parce que les réactions du corps social entier vont de la demande de protection sociale à la montée des totalitarismes, en passant par le repli protectionniste. Il faut ainsi comprendre le développement de l'interventionnisme public et notamment étatique au cours des trois premiers quarts du XXe siècle comme un processus de « ré - encastrement» visant à restaurer les conditions de vie en commun.

Il est clair que ces tendances et contre - tendances ne concernent en aucune manière l' «encastrement - étayage », dont les modalités peuvent certes changer dans le temps et dans l'espace, mais qui n'est pas susceptible d'avancées ou de reculs. Les processus de « désencastrement - insertion » se sont du reste affirmés historiquement, non pas dans un vide social, mais bien au contraire, au travers de changements institutionnels tels que, lors de la Révolution Industrielle en Angleterre, les Enclosures et la loi sur les pauvres de 1834.

\footnotetext{
${ }^{2}$ Les activités économiques chez Polanyi ne relèvent pas d'une définition formelle (optimisation de l'usage de moyens rares) mais d'une définition substantive (activités dérivées de la dépendance de l'homme vis-à-vis de la nature et de ses semblables). Polanyi distingue notamment trois modes d'intégration ( la réciprocité (le don), la redistribution et l'échange marchand.
} 
Toutefois les deux définitions sont en nécessaire interaction, comme le remarque encore $\mathrm{R}$. Le Velly (op. cit.). Le simple fonctionnement des activités marchandes suppose la confiance et la présence de règles du jeu communément acceptées. Les modalités d'étayage peuvent ainsi être en écho avec le degré et les modalités d'insertion de l'économique dans le social. Il y a alors renforcement mutuel, comme dans le cas d'une culture de marché qui en facilite le fonctionnement. Mais le cas inverse, plus intéressant pour notre propos, survient lorsque « les contraintes qui émergent des ordres marchands entrent en conflit avec les conditions d'encastrement sur lesquelles s'appuient ces ordres » (op. cit. p. 193). C'est en particulier ce que Polanyi a repéré dans les exigences « inhumaines » de création d'un « véritable » marché du travail dans l'Angleterre de la Révolution Industrielle, la rationalisation marchande avançant au prix de la déstabilisation des communautés qu'elle affecte. Nous voyons ainsi apparaître un risque d'incohérence foncière de nos sociétés dotées de marchés très développés, et c'est alors que l'on peut comprendre la dramatisation inhérente au concept de « désencastrement » tel que Polanyi l'a forgé. Celle-ci peut bien être mitigée en observant que les marchés sont des institutions très anciennes et qu'ils ont parfois été développés à très grande échelle avant le capitalisme ; ou encore en observant que l'encastrement - insertion est susceptible de multiples degrés. Il n'en reste pas moins que l'élaboration polanyienne est marquée par des basculements, tendances et contre - tendances, et non par des gradations.

I.2. L'encastrement / désencastrement, à la base de la variété des régimes de protection sociale selon Esping - Andersen.

C'est peut-être dans le domaine des réponses, en seconde moitié du XXe siècle, aux menaces du marché autorégulateur que le concept d'encastrement s'est révélé le plus fécond, bien après les formulations de K. Polanyi. On peut en effet trouver dans les travaux du politologue danois Esping - Andersen un usage systématique de la dualité «marchandisation » - « dé marchandisation » dont il marque l'origine chez Polanyi (Esping - Andersen 1990, p. 3 et 36) Celui-ci cherche à analyser les diverses modalités de l'Etat - providence national, qui est, comme on le sait, le cœur de la réponse en termes de protection sociale et de stabilisation des marchés que Polanyi anticipait dans La grande transformation. Esping - Andersen se focalise sur les choix qui organisent la protection sociale et isole deux dimensions clés. L'une est la place, centrale ou résiduelle, laissée aux mécanismes marchands dans les activités qui conditionnent la vie des personnes et notamment les tâches domestiques, l'éducation, le soin des enfants... Ce qu'il appelle « commodification » et « de - commodification ». Tout en reprenant directement les fondements de l'idée d'encastrement - désencastrement, cette nouvelle dénomination introduit une nuance. Il s'agit d'un processus politico - institutionnel susceptible de gradations, les responsables politiques pouvant en quelque sorte choisir la position du curseur marché - non-marché. Esping - Andersen s'emploie ainsi à construire empiriquement, pour divers pays, des scores de « de - commodification ». L'autre est la place accordée à la famille en tant qu'institution pouvant fournir en tout ou partie ces services et former un noyau de solidarité plus ou moins actif. Alors on débouche sur une typologie célèbre, qui oppose les régimes «nordiques » et «anglo-saxons » ou «libéraux »: les premiers mettent en place un Etat - providence très développé et laissent peu de place au marché ; ils favorisent l'individualisation des droits et des obligations, limitant par là même le rôle de la famille en tant qu'institution. Les seconds en revanche limitent la taille et le rôle de l'Etat redistributeur et protecteur, assurant une large place aux relations de marché (par exemple pour des crèches), tout en limitant eux aussi le rôle de la famille. On trouve alors 
dans une position intermédiaire les régimes dits «continentaux » et «méditerranéens », qui s'appuient à la fois sur un Etat redistributeur et protecteur moyennement développé (ne couvrant pas toute la population au même degré) et sur l'institution traditionnelle de la famille (l'homme assurant normalement la fonction de «breadwinner») à laquelle est attribué un grand rôle. Le grand apport de cette typologie est d'abord de saisir la variété des réponses aux défis du marché autorégulateur, qui déclinent et combinent, à des degrés divers, la place et le rôle de l'Etat et des activités familiales. Il est ensuite dans l'introduction au cœur des choix collectifs de la dimension des inégalités entre hommes et femmes.

Au regard du concept d'encastrement / désencastrement et des préoccupations polanyiennes, on note alors un déplacement de la dramatisation. En effet, l'analyse d'Esping - Andersen souligne la cohérence des choix «nordiques », mais aussi «anglo - saxons », les premiers créant un haut niveau de prélèvements sociaux pour aboutir à un haut niveau de solidarité et à une offre riche de services collectifs, et les second faisant l'inverse : peu de prélèvements, peu de redistribution, et une large place laissée à l'initiative individuelle sur les marchés. Ces régimes s'accommodent ainsi d'un niveau d'inégalités très supérieur à celui que tolèrent les pays nordiques. Mais surtout l'auteur montre aussi l'instabilité qui devrait affecter les régimes « continentaux » ou «méditerranéens ». Ceux-ci, d'obédience Bismarckienne, sont dans une position intermédiaire du point de vue des inégalités. Ils réservent à certains groupes (de salariés stabilisés) un niveau élevé de protection, et en excluent d'autres, condamnés à des emplois instables et à des miettes de services collectifs. Ils sont donc porteurs de conflits et de déchirures. Et ils reposent sur un modèle de solidarité familiale cantonnant les femmes aux activités domestiques ou aux métiers d'appoint, modèle qui deviendra vite obsolète à mesure que celles-ci pourront s'émanciper.

Ce diagnostic d'instabilité prend une force accrue dans le contexte actuel d'expansion à nouveau de la sphère financière et d'interconnexion des marchés à l'échelle de la planète. En effet, les risques de concurrence fiscale et de délocalisation mettent à l'épreuve les régimes de protection sociale, la solution la plus simple aux difficultés de financement étant l'extension du rôle reconnu aux relations de marché, compensée, pour les plus défavorisés, par la mise en place d'un filet de sécurité minimal. On assiste alors à trois évolutions différenciées : un renforcement de la lutte contre la pauvreté dans les régimes «libéraux », un recalibrage des régimes «nordiques », ceux-ci étant en quelque sorte amincis, étendant à la marge le rôle dévolu aux marchés, et une mise en cause plus radicale des régimes «continentaux", confrontés au rétrécissement de leur base de financement au moment où se multiplient les besoins.

Sans entrer ici dans une appréciation critique de l'apport de cette analyse célèbre, dont le gain de réalisme opérationnel est indiscutable, on notera quelques interrogations. D'abord les régimes Bismarckiens peuvent élargir leurs bases de financement et se lancer dans des processus d'hybridation (Gazier et Lechevalier 2006). La gamme des choix qui leurs sont ouverts ne se limite pas à l'extension du domaine marchand ni à la convergence avec le régime «anglo - saxon ». Ensuite, et dans la ligne directe des priorités polanyiennes, on observe que l'avancée (ou le recul) du marché est ici principalement compensée par le recul (ou l'avancée) de l'Etat social, ce qui ne laisse guère de rôle explicite aux organisations non marchandes ni à l'économie sociale. Sans doute ce partage trop simple entre marché et Etat est-il une des sources du statisme qu'il est aisé de reprocher aux catégorisations d'Esping Andersen. Plus généralement, on peut s'interroger sur le jeu de compensations ou d'équilibrage qui s'instaure entre les initiatives publiques, privées marchandes et privées non marchandes. Par exemple, les activités de soins aux personnes tels que la garde et l'éducation 
d'enfants (le «care ») peut être assurées simultanément par l'Etat (au moyen de crèches publiques), moyennant paiement par des individus offrant ces services, par des organisations marchandes ou non marchandes subventionnées ou non, ou encore gratuitement par une personne de la famille étroite ou élargie. Des innovations récentes ont du reste remis en cause et commencé à dépasser cette typologie : par exemple des entreprises collectives fournissant des services de garde, dirigées par des comités de parents et des travailleurs - formateurs, bénéficiant d'un contrat de services avec l'Etat, avec un très fort subventionnement qui leur permet d'offrir des tarifs très bas. L'expérience des Centres de petite enfance au Québec en est une illustration. La question clé devient celle de la viabilité de la combinaison retenue, et des conséquences sur les conditions de vie et l'autonomie des divers groupes sociaux concernés.

\section{I.3. Une seconde «grande transformation »? Avatars et mutations du ré - encastrement}

Sans entrer dans les différenciations internes aux pays capitalistes développés, mais en gardant à l'esprit les complexités qu'elles rendent manifestes et condensent, il est possible de synthétiser aujourd'hui, en termes largement polanyiens, les processus qui mènent à la vague de désencastrement des années 1980 et 1990, pour s'interroger sur le contenu de nouvelles réponses visant à un «ré - encastrement ». C'est ce que propose l'analyse de B. Lévesque (Lévesque 2003). Il résume deux cents ans de l'histoire occidentale termes de défaillances successives: une évolution heurtée a mené de la prééminence de la société civile traditionnelle - avec les défaillances des corporations et de l'ordre stratifié et figé de l'ancien régime -, à l'essor du marché - avec la défaillance d'une "société ou prédominaient la dépendance des personnes et la confusion des rapports politiques et des activités économiques » (ibid, p. 497) -, puis à l'entrée en scène de l'Etat protecteur, celui-ci trouvant désormais ses limites. C'est le couple Etat - marché, dont le fonctionnement doit beaucoup à la doctrine keynésienne, qui est désormais en crise dans le contexte de la "globalisation » et de l'émergence de nouveaux rapports de production et de consommation associant flexibilité, mise en réseau, innovation et exigences de participation à tous les niveaux.

Dans cette grande dynamique, deux voies s'ouvrent alors. L'une est le retour à davantage de marché, en prenant appui notamment sur la critique de la lourdeur, de la bureaucratie et de l'opacité de l'Etat. On risque alors, en tentant d'instaurer à nouveau un « marché célibataire », de retrouver des risques et dysfonctionnements proches de ceux qui avaient été expérimentés de 1830 à 1950. L'autre consiste à faire revenir au premier plan la société civile, dans une acception renouvelée, celle qui promeut la logique de l'engagement volontaire et l'adhésion à un projet commun. Mais cette logique, que B. Lévesque identifie comme composante essentielle d'un « ménage à trois » Etat - marché - société civile, ne connaîtrait-elle pas elle même des défaillances, et à quelles conditions peut-elle s'imposer ?

Nous nous trouvons ainsi face au débat contemporain sur les composantes et le fonctionnement de l'économie mixte. Il n'est évidemment pas question, dans le cadre de cet article, de prétendre l'examiner en détail. La tâche ici pertinente est de suivre le fil polanyien, en posant une interrogation globale : comment s'organise la maîtrise de la société sur les conditions de vie de ses membres ? Au moins deux gammes de réponses, complémentaires, sont possibles: l'une est pragmatique, et consiste à revenir directement sur le jeu des défaillances, pour chercher comment sinon le stabiliser, du moins l'organiser. L'autre, plus ambitieuse, cherche à identifier, en positif, le contenu nouveau de l'économie mixte qui se dessine au travers des transformations en cours. 
Les travaux d'A. Ben - Ner et T. Van Hoomissen (par exemple Ben - Ner et Van Hoomissen 1991) illustrent la première démarche. Ils proposent un circuit fictif de choix possibles organisés en séquences. Le point de départ est la défaillance du marché dans la réponse à un besoin collectivement ressenti, par exemple la fourniture d'un service collectif. Alors la première réponse possible est celle de la production par l'Etat, qui elle-même est susceptible d'être insatisfaisante. Alors on peut envisager de recourir à l'économie non marchande, qui elle - même peut satisfaire ou non le besoin. L'apport des auteurs est notamment d'amorcer la réflexion sur la pertinence et l'efficacité comparative de ces trois voies, de différencier les coalitions qui portent ces divers arrangements, et de réintroduire la défaillance du non marchand dans le circuit des décisions. Le prolongement de la démarche se lit dans les travaux ultérieurs d'A. Ben - Ner (Ben - Ner 2006), qui adoptent une perspective en termes de gouvernance et de coûts de transaction. Distinguant classiquement pour l'Etat la fourniture directe par entreprises publiques ou indirecte par appels d'offre et la réglementation, ces recherches proposent des clés de compréhension des mouvements de frontière entre les trois sphères du public, du privé marchand et non marchand, tant dans l'espace (entre pays) que dans le temps.

Directement opérationnelle, cette démarche micro - économique et sectorielle conjugue finesse et réalisme, sans toutefois s'interroger sur les conditions plus globales du jeu conjoint des trois sphères, ni sur les conditions politiques et sociales des choix entre elles pour la réalisation d'une activité donnée. La place de l'économie non marchande ne dépend-elle que de son efficacité comparée et de la plus ou moins grande facilité de l'accès au financement ? La fresque tracée par B. Lévesque (op. cit.) explore une autre voie en suggérant que l'économie mixte d'aujourd'hui est elle - même en mutation. La combinatoire public, privé marchand et non marchand voit ses modalités de fonctionnement mais aussi ses priorités subir un processus de redéfinition. Partant des changements affectant la protection sociale, notamment des exigences accrues de participation et de responsabilisation, B. Lévesque reprend les travaux (par exemple Giddens 1999) qui voient s'affirmer un Etat - providence positif, actif voire «activant». Celui - ci ne connaît toutefois pas une évolution univoque : il est pris dans l'alternative entre le retour néo - libéral à plus de marché et plus d'initiative individuelle, via le «workfare» et un ciblage accru des politiques publiques, et des perspectives «progressistes» qui privilégient l' «empowerment» des personnes et des collectivités, le niveau local et la diversité des acteurs sociaux. Au centre de cette mutation indécise, se trouvent l'accroissement conjoint de droits et de responsabilités, le passage d'une culture de la dépendance à une culture de l'autonomie et de la réciprocité, et finalement un glissement dans la conception même des mesures sociales, qui tendent à devenir des investissements sociaux.

Ces transformations entrent en interaction avec d'autres qui affectent en parallèle la sphère économique. B. Lévesque les synthétise en mentionnant l'apparition d'une "offre intégrée » venant compléter et dépasser la gestion par la demande typique de l'ère keynésienne. L'Etat devient, face à la nécessité de garantir des infrastructures et de favoriser l'innovation et l'apprentissage, un Etat partenaire voire un Etat catalyseur. Ses priorités deviennent de rendre possible ou de stimuler les activités de $\mathrm{R} \& \mathrm{D}$, la formation de la main - d'œuvre, l'accès aux financements. Cette nouvelle économie mixte, qui conjugue mises en réseau et spécialisations, doit ainsi faire émerger, par exemple, une meilleure synergie entre les secteurs capitalistes et l'économie sociale. On retrouve alors le social comme investissement, et non pas comme frais supplémentaires nécessaires à l'acceptation sociale des changements économiques. Dans cette perspective, les dépenses pour améliorer l'environnement et la 
qualité de vie, les investissements dans le capital social affirment alors leur rentabilité. Investir dans les « ressources humaines », dans les enfants et dans les compétences, apparaît comme la clé d'une économie durable et innovante.

Bouclons la boucle comme nous y invite B. Lévesque lui-même (ibid. p. $503-4$ ) : cette redéfinition tendancielle conjointe de l'économique et du social se rapproche de la définition même de l'économie «substantive » telle que l'avait posée Polanyi dans The Livelihood of Man (Polanyi 1977 et 2007). Celle - ci ne se limite ni au marchand ni au monétaire, et inclut ainsi au premier chef les activités de l'économie sociale. Par des cheminements complexes, l'étude des processus actuels de désencastrement et de réencastrement conduirait à un déplacement de la pertinence du propos polanyien. Elle serait moins dans la dénonciation globale de la «marchandisation » des activités, puisque les combinatoires de l'économie plurielle se révèlent complexes et évolutives, que dans le nécessaire renforcement réciproque de l'économique et du social. Evitant de verser dans un optimisme final qui serait singulièrement inadapté en notre début de siècle inégalitaire et instable, c'est par une discussion du le rôle d'impulsion et de garantie de l'Etat, et sur les apports de la démocratie délibérative que conclut cette contribution particulièrement suggestive de $\mathrm{B}$. Lévesque.

Nous aurons l'occasion de reprendre ces thèmes, mais à ce stade de notre réflexion, trois observations s'imposent sur trois discussions complémentaires qu'elle appelle. On peut d'abord se demander si la fresque ne se clôt pas sur une représentation dialectisée trop globale et finalement fusionnelle, la dynamique des déchirures et des désencastrements débouchant sur une perspective réconciliatrice dans laquelle les investissements «sociaux » seraient a priori et généralement d'une forte rentabilité et feraient aisément la preuve de leur utilité incontournable. Ensuite, la question de l'égalité et de l'inégalité motiverait d'autres développements. Les développements actuels de l'Etat - providence actif semblent promouvoir, via l'affirmation de la responsabilité individuelle, des valeurs d'équité admettant des écarts à l'égalité. Quels accroissements de l'inégalité faut-il admettre ? Ne s'agirait-il pas plutôt de rechercher une redéfinition de l'égalitarisme plutôt que d'admettre son recul ? De même, la question de la détermination de l'intérêt général n'est qu'effleurée, et renvoyée à une délibération collective. Cette issue procédurale a toutefois l'intérêt de focaliser l'attention sur la démocratisation comme enjeu polanyien majeur et méconnu.

\section{I.4. Incohérences et changement institutionnel}

On rejoint alors la question du changement institutionnel, lorsque coexistent le noyau central d'un modèle économique et social, et une série de couches périphériques qui en sont de plus en plus éloignées (Radice 2000). Le noyau est formé d'acteurs qui se conforment strictement à la logique centrale, cependant que les couches périphériques obéissent à des logiques plus diversifiées. Par là - même, elles remplissent plusieurs fonctions face aux pressions venues de l'extérieur et qui sont susceptibles de perturber, voire de remettre en cause le fonctionnement central. Elles peuvent en effet absorber les pressions en faveur du changement, et par là protéger voire légitimer le modèle existant. Les mouvements des acteurs périphériques vont toutefois au-delà de l'absorption des chocs et de l'adaptation passive. Ils peuvent innover plus aisément que les acteurs centraux, et ainsi déclencher des processus d'adaptation active et créatrice, ce que l'on peut appeler l'innovation sociale. Alors les acteurs centraux se trouvent devant un dilemme : ou bien réaffirmer envers et contre tout la logique initiale mise en cause à la périphérie, qui à la fois absorbe et innove. Ou bien initier un processus de changement 
radical, coûteux et incertain parce qu'il met directement en cause la logique initiale. Il résulte de cette schématisation du changement par la périphérie, l'affirmation d'une inévitable instabilité stratégique au niveau du centre. C'est une première dimension de l'incohérence systémique, qui marque les choix des institutions centrales lorsqu'elles font face à des remises en cause d'origine externe.

Il en résulte que les effets des innovations sociales sont toujours difficiles à évaluer, parce qu'ils débordent des problèmes qu'elles affrontent directement, pour mettre en cause les relations de pouvoir au centre même du système. Elles sont souvent locales, peuvent naître par exemple dans le cadre d'un territoire ou d'un secteur. Les impacts peuvent se propager doublement : horizontalement entre sous - systèmes plus ou moins reliés, et verticalement lorsqu'ils affectent les équilibres macro-économiques ou les pratiques gouvernementales.

Appliquer cette vision du changement aux transformations du capitalisme actuel dans la «globalisation » peut ainsi déboucher sur un diagnostic en termes de crises des régulations nationales et même d'éclatement. Il a été proposé il y a déjà dix ans par des auteurs tels que Hollingsworth et Boyer (1997), qui retrouvent une fois encore et transforment le concept d'encastrement, pour proposer celui de «nestedness». Ces auteurs désignent par là la dispersion des arrangements institutionnels, opérant à de multiples niveaux spatiaux (locaux, régionaux, continentaux...), qui caractérise les multiples processus d'adaptation à la mondialisation, déclenchés sans souci de cohérence par des acteurs faiblement coordonnés. La prolifération de ces «niches » d'innovations pose alors la question de la complexité institutionnelle, ou plus simplement celle du désordre.

Dans la perspective de Karl Polanyi, le désordre est plus grand encore parce qu'il identifie une seconde source d'incohérence, interne au modèle ainsi mis sous pression. Il insiste en effet sur le paradoxe central du système actuel: sa viabilité dépend en permanence d'interventions dont, par son orientation même, il prétend se passer. La logique du désencastrement tend à séparer l'économique du social, mais la mise en œuvre réelle de cette séparation serait mortelle à son fonctionnement; elle génère ainsi des interventions continuelles pour pouvoir continuer à s'exprimer tout en étant compensée. Cette contradiction interne est ainsi créatrice au sens de la destruction créatrice de Schumpeter. Alors les initiatives de la périphérie voient leur potentiel et leur signification se renforcer. Elles constituent en effet des « alternatives libératoires » (Harvey 2000, p. 186) qui, en dépit de leur diversité et de leur non - coordination initiale, peuvent s'affirmer doublement au sein du système dominant. D'une part elles peuvent se constituer en réseaux et tendre à s'institutionnaliser; la phase des «niches » ne devrait donc avoir qu'un temps. D'autre part elles rendent plus visibles les incohérences et les insuffisances du modèle central initial. La perspective devient ainsi, paradoxalement, plus optimiste. La possibilité que les acteurs périphériques restent marginalisés, et ne servent qu'à compenser partiellement les insuffisances du centre, ou encore qu'ils soient conduits, par mimétisme ou sous la pression, à reconduire un «isomorphisme institutionnel », n'a pas disparu. Mais elle n'est pas la seule voie prévisible ni même la plus probable. On peut donc s'intéresser aux modalités de transmission des innovations institutionnelles.

Sur ce point, l'œuvre entière de Polanyi serait à prendre en compte, parce qu'elle apporte une série d'éléments qui débordent et encadrent les développements de La grande transformation. Parmi eux, se trouvent d'abord, comme on le sait, l'accompagnement de l'expérience du « socialisme municipal » de «Vienne la Rouge» $(1918$ - 1934). Elle l'a conduit à formuler la proposition d'une «démocratie fonctionnelle» qui représente selon lui l'essence du 
socialisme ( «living essence of socialism ») et à se consacrer pendant plusieurs années au projet d'une «théorie positive de l'économie socialiste ». Il préconisait un modèle associatif décentralisé en sorte que l'efficacité de chacun dans sa double identité de producteur et de consommateur se conjugue avec un engagement en faveur du bien - être collectif, les associations créent un lien entre le niveau individuel et le niveau collectif, et assurant une vision d'ensemble, enclenchant un processus d'apprentissage collectif.

Mais par la suite les priorités de Polanyi se sont déplacées vers une série de débats et de projets portant sur l'éducation au Royaume - Uni (Mendell 1994). Anticipant sur le thème désormais bien connu de la «construction sociale de la connaissance », et convergeant avec des travaux tels que ceux de Paolo Freire sur la «pédagogie de la libération », ses réflexions sur «l'éducation socialiste» ont exploré comment une pédagogie enracinée dans la réalité vécue peut à la fois être efficace pour l'acquisition et l'appropriation de savoirs et de pratiques, et dans la remise en cause du modèle dominant. Ici encore il repère les voies d'une transformation économique et institutionnelle, par l'accent mis sur la réappropriation de la connaissance.

«Démocratie fonctionnelle » et «politique de la pédagogie», ces deux cheminements sont ceux que reprend aujourd'hui, sur une autre base, le développement de l'économie sociale et de ses diverses formes d'institutionnalisation. Ces ambitions sont par nature plus sectorielles, et ceci avive les tensions internes et externes : tout d'abord, combien de temps peut-on s'accommoder d'une multitude d'expériences fragmentées avec leurs spécificités souvent nationales (même la nomenclature est révélatrice : le tiers secteur, le tiers système, l'économie sociale, l'économie solidaire, les entreprises sociales, les « Non Profit Organisations », etc.) alors que les visées sous-jacentes sont générales?

D'autre part, le circuit des possibles défaillances généralisées (du marché ; de l'Etat; et de l'économie sociale) peut générer un scepticisme aux couleurs d'un pseudo - pragmatisme. Il faudrait développer «ce qui marche », même au prix d'entorses aux principes fondateurs. Une telle démarche risque d'affaiblir l'économie sociale sous couvert de la renforcer. Ceci débouche sur une troisième série de tensions, celles qui portent sur le repérage et la mesure de l'efficacité des initiatives «sociales » au regard des activités marchandes, et apparaissent parfois comme des courts-circuits logiques : quelle est l'efficacité d'un réseau de bénévoles ? y a-t-il un intérêt du désintéressement ? une performance du don ?...

Ces tensions doivent alimenter une dynamique sociétale démocratique, non pas pour y être résolues ou supprimées, mais relayées et élaborées. La vision de la démocratie qui découle de ces analyses fait logiquement une place majeure aux conflits, autant dans les idées que dans les intérêts. En complément des orientations polanyiennes, on peut ainsi placer la conception selon laquelle la « démocratie délibérative » vient doubler la démocratie politique d'un espace public «agonistique » (C. Mouffe 2000). Entre tensions et négociations, la mise en cause des hiérarchies instituées est la conséquence permanente des tendances au désencastrement et des contre - tendances qu'elles génèrent. 


\section{Stratégies du réencastrement}

Rien ne serait plus éloigné de la pensée de K. Polanyi que la perspective d'un menu de politique générale dosant à volonté les parts respectives du marchand et du non-marchand. Mais il était convaincu de la nécessité de combiner la coordination marchande avec les autres modalités d'échange et d'interaction que sont la réciprocité et le don, et la redistribution, et cette conviction trouve comme on l'a vu une nouvelle actualité en ce début de siècle. C'est donc en termes de contrôle des conditions de vie, et de stratégies assurant et faisant évoluer ce contrôle qu'il faut reprendre la perspective polanyienne. Il en résulte, sur un plan très général, des liens forts avec une série de projets et de théories qui insistent, avec des priorités et des angles d'attaque divers, sur la promotion de l'initiative et de la délibération individuelles et collectives. L'œuvre entière d'Amartya Sen, avec sa focalisation sur les « capabilités » et sur la construction sociale de la «liberté réelle»s'inscrit ainsi, à partir d'un point de vue principalement microéconomique, en prolongement direct de cette orientation.

Ce sont les conditions de mises en œuvre qu'il convient ici d'interroger, dans leurs liens à l'emprise du marché. Les stratégies de ré-encastrement sur lesquelles nous allons nous focaliser peuvent d'abord être abordées en termes de contrôle d'un certain nombre de ressources et d'outils productifs, c'est alors la problématique de l'économie sociale dans sa capacité à développer et diffuser des innovations sociales. Pour en analyser la portée générale, nous nous centrerons sur l'expérience québécoise récente en lui appliquant un concept postérieur à K. Polanyi mais directement inspiré de ses travaux : le «processus institué de démocratisation économique ». (Mendell 2007 a et c) Hormis le Québec et le Pays Basque, les développements de l'économie sociale dans d'autres expériences nationales sont souvent consistants, mais restent séparés. A quelles conditions peut-on espérer qu'ils pèseront sur le fonctionnement d'ensemble de l'économie et de la société ? Deux stratégies transversales, très différentes, proposent des éléments de réponse complémentaires. L'une s'intéresse au contrôle du marché du travail, névralgique pour Polanyi puisqu'il gouverne le devenir matériel d'une grande majorité de la population dans les pays développés. Elaborés en Europe, les travaux menés en termes de «marchés transitionnels du travail » mettent en avant la double nécessité d' "équiper les gens pour le marché », mais aussi d' "équiper le marché pour les gens », renversement très polanyien qu'il est intéressant d'expliciter. La seconde stratégie, explorée aux Etats-Unis et dans les grandes organisations internationales, revient au plus près de la théorie économique standard, pour explorer les conséquences de l'idée de «bien public ». Une série de développements récents ont pu baliser un domaine de nouvelles priorités collectives à côté des anciennes, au sein duquel les transformations récentes du rôle de l'Etat peuvent être mises en évidence et questionnées. Il est enfin possible, en gardant en tête ces élaborations transversales, de préciser la place que peut occuper aujourd'hui le socialisme associationniste de K. Polanyi dans l'ensemble des doctrines du «socialisme libéral ».

II.1. L'expérience québécoise, vers un «processus institué de démocratisation économique »

Au-delà de l'économie sociale comme simple modèle organisationnel, c'est finalement sa capacité de transformation qu'il convient d'examiner, dans ses conditions et ses modalités. Si efficace soit-il dans sa réponse à des besoins non satisfaits, le développement sectoriel risque 
de s'enfermer dans une forme de dépendance compensatrice s'il ne parvient pas à se structurer pour enclencher, dans l'ensemble de l'économie et de la société, un processus de changement institutionnel délibéré. Le «processus institué de démocratisation économique » (Mendell 2007a et c) désigne ainsi le cheminement par lequel les acteurs de l'économie sociale s'engagent dans un dialogue avec l'Etat pour «co-construire » avec lui de nouvelles politiques publiques. Ce dialogue, pour être établi et équilibré, suppose l'unification des diverses organisations d'économie sociale dans la formulation et la négociation de politiques alternatives.

L'expérience québécoise depuis les années 1980 permet de retracer un tel cheminement. Elle montre en effet comment des initiatives diverses, en réponse aux défis que suscitent les crises financières et d'emploi accompagnant la «mondialisation», ont débouché sur la création d'organisations intermédiaires, associant de multiples parties prenantes entre l'Etat et la société civile, dans les années 1980, et sur une structuration en «réseau de réseaux» permettant la création et l'aménagement d'espaces institutionnels spécifiques. La date charnière à cet égard est 1996, lorsque fut créé un tel «réseau de réseaux »: le «Chantier de l'économie sociale », dans le but de constituer en force politique et partenariale la multiplicité des organisations d'économie sociale.

Il s'agit là de la maturation de pratiques anciennes. On peut en effet considérer que quatre générations d'économie sociale se sont succédé au Québec depuis les années 1850. Durant ces premières années, ont été créées des mutuelles de travailleurs. A partir des années 1900, on observe l'apparition des coopératives agricoles et du mouvement des caisses populaires. Ce premier élargissement s'amplifie dans les années 1930 : apparaissent alors des coopératives forestières, dans le bâtiment, l'éducation et la consommation. Elles se structurent dans le Conseil Supérieur de la Coopération du Québec dès 1939. La quatrième génération date quant à elle des années 1960. La mobilisation des mouvements sociaux pour une démocratie plus participative a donné lieu à de nombreuses initiatives mises en œuvre par la société civile en santé, services sociaux, éducation populaire, entre autres. Mais à partir des années 1980, face aux restructurations qui bouleversent le tissu productif et déstabilisent ses travailleurs, il s'est agi de passer de l'intervention sociale à l'intervention économique. Les objectifs de revitalisation locale, de création d'emploi, d'intégration et de lutte contre la pauvreté, conduisent à affirmer la nécessité d'une «économie négociée » faisant leur part à l'ensemble des parties prenantes. La création d'intermédiaires et d'espaces de négociation a donné lieu à une meilleure coordination horizontale entre des structures très diverses, et à une intégration des politiques. Les interventions affectant le marché du travail doivent ainsi être coordonnées avec la fourniture de services aux entreprises de manière à stimuler la revitalisation locale et la prise d'initiative économique. Non seulement la société civile est présente dans ces nouveaux lieux de négociation donnant lieu à une gouvernance nouvelle, mais aussi ses acteurs jouent un rôle clé en tant qu'architectes de ces nouveaux espaces publics décisionnels.

Ces élargissements successifs ne sont pas le résultat d'un processus harmonieux et pacifié, mais témoignent de tensions, de conflits et d'apprentissages, par lesquels les acteurs travaillent ensemble sur des objectifs communs pour aller, au-delà de l'intérêt collectif, vers l'intérêt général. Le choix d'unifier les organisations d'économie sociale dans un "réseau de réseaux », qui incorpore les secteurs d'activité ainsi que les mouvements sociaux et les regroupements des organisations de développement, est une réponse à la complexité qu'induit leur variété et leur dispersion, et au défi d'acquérir un véritable pouvoir de négociation au delà des territoires spécifiques. La capacité à parler d'une seule voix renforce le pouvoir des membres du réseau et permet la co-construction de politiques publiques elles-mêmes 
favorables aux initiatives individuelles et aux projets collectifs. Ce processus renforce aussi la reconnaissance de l'économie sociale comme un acteur économique incontournable au Québec. Ceci étant dit, une série de barrières institutionnelles apparaissent, qu'il faut surmonter. Les lois, les normes comptables par exemple, ignorent souvent les spécificités de l'économie sociale. Malgré sa reconnaissance, elle a par ailleurs une image marginale et exclusivement non marchande, et elle souffre fréquemment, sur le plan strictement économique, d'une sous-capitalisation financière. Les réponses explorées sont elles-mêmes multiples mais leur ensemble tend à constituer un système d'innovation. Il combine notamment la création de fonds d'investissement pour mieux doter les entreprises en capital, la formation des travailleurs et l'analyse du marché du travail, et encore la création de marchés pour les biens et les services de l'économie sociale, avec des pratiques de labellisation et l'organisation de foires. Le projet du «Chantier de l'économie sociale» cherche aussi à capitaliser les leçons des expériences tant positives que négatives, notamment en analysant et diffusant systématiquement les informations sur ces expériences. Ce foisonnement s'observe au milieu de tensions aussi bien internes qu'externes. La coexistence entre l'économie sociale, l'économie marchande et les politiques publiques devient ainsi un objet de négociation.

La stratégie de ré-encastrement passe ici par la constitution d'un système d'innovation dont la dynamique est à la fois interne et externe. En interne, il s'agit de prendre appui sur les réalisations de l'économie sociale et notamment ses apports à l'économie territoriale, pour en fortifier le développement en faisant agir ensemble de nombreux composants, qui vont de l'intervention de fonds d'investissement à des politiques de formation de la main-d'œuvre en passant par le conseil aux entreprises et bien sûr la recherche. En externe, il s'agit d'influencer les politiques étatiques, de manière d'abord à obtenir une reconnaissance et une stabilisation des institutions et des organisations de l'économie sociale, mais aussi de faire évoluer la détermination et l'appropriation des politiques publiques. Par la création d'espaces publics de débat et de concertation, le but est bien de modifier les fonctionnements traditionnels de la gouvernance publique, en passant d'une logique « top down » à une logique plus horizontale. On rejoint alors toute une littérature consacrée aux gains collectifs qui résultent de la création d'espaces publics associant les parties prenantes et de l'organisation de liens horizontaux entre les départements ministériels (Sullivan et Skelcher, 2002).

Cette stratégie complexe et ambitieuse se distingue du développement séparé d'un tiers secteur tel qu'on peut l'observer aux Etats-Unis, tout comme de l'adossement à des entreprises publiques tel qu'on peut l'observer dans de nombreux pays européens. Elle indique une voie séduisante. La complexité de cette intégration pose évidemment la question des conditions de sa généralisation. D'un côté, on peut faire valoir que l'utilisation et la mise en valeur directe des solidarités de proximité sont une composante inévitable de tout processus de ré-encastrement. De l'autre, on peut conjecturer que, hors contexte spécifique et longuement préparé, le dépassement de l'horizon multisectoriel restera encore, dans un futur prévisible, un défi posé aux acteurs de l'économie sociale. Mais d'autres stratégies de réencastrement peuvent s'envisager, s'appuyant sur des logiques transversales.

II.2. Réencastrer le marché du travail : les «mobilités protégées » et les « Marchés Transitionnels du Travail ». 
Issue de travaux menés à Berlin dans les années 1990, la perspective des «Marchés Transitionnels du Travail »(MTT) s'est d'emblée centrée sur le destin des chômeurs de longue durée, ceux-ci subissant une exclusion sur le marché du travail. Prenant acte des échecs fréquents des politiques de l'emploi traditionnelles, elle recherche les conditions de la réinsertion de ces travailleurs dans un réaménagement global des «transitions » qui ont lieu sur le marché du travail et autour de lui (Schmid et Gazier (dir) 2002). Dans leur acception originale, les «transitions » désignent tout écart à la norme de l'emploi durable à temps plein. On distingue notamment les transitions au sein de l'emploi (par exemple entre temps plein et temps partiel), entre éducation et emploi (par exemple l'apprentissage), entre chômage et emploi (on retrouve alors les politiques de l'emploi), entre emploi et activités domestiques (par exemple un congé parental ou sabbatique), entre emploi et inactivité (par exemple un passage progressif à la retraite). Tels sont les cinq champs principaux qui sont en interaction et qu'il s'agit de considérer dans leur dépendance réciproque ${ }^{3}$.

«Ma mobilité dépend de la vôtre »: cette intuition systémique est à la base d'analyses et de propositions visant à restaurer un meilleur contrôle collectif sur le marché du travail. La préconisation centrale des MTT est de promouvoir l'aménagement systématique et négocié des «transitions », laissant le libre choix aux personnes mais aménageant l'espace de leurs choix de manière à leur donner un pouvoir effectif de décision («empowerment») et à organiser la solidarité collective. C'est ainsi que face au chômage de masse, des dispositifs tels que la «rotation des emplois » utilisés au Danemark lors de la seconde moitié des années 1990 ont pu combiner des départs en congés formation avec l'ouverture de postes temporaires pour des chômeurs, ceux-ci étant très fréquemment conservés lors du retour en entreprise du travailleur ayant pris le congé. Les chômeurs non conservés reprenaient alors leurs recherches sur le marché du travail, mais avec de bien meilleures chances de réembauche puisqu'ils pouvaient faire valoir une expérience récente de travail en entreprise.

Cet exemple isolé et temporaire a l'intérêt de montrer en quoi les dépendances réciproques entre «transitions » peuvent être exploitées, ici dans un processus souple et adaptable de partage intertemporel du travail et de ré-homogénéisation de la main-d'œuvre (les uns se formant pendant que les autres les remplacent au travail, et la collectivité bénéficiant à terme de travailleurs mieux formés et plus employables). Le débouché central de ces préconisations se trouve dans la mise sur pied de négociations sur les «transitions» associant, principalement sur une base régionale ou locale, les partenaires sociaux, les collectivités locales et les réseaux associatifs et non marchands. Ce cadre permet ainsi d'articuler explicitement l'économie sociale et son offre de postes durables ou temporaires, à l'ensemble des positions professionnelles ou domestiques généré par le tissu des entreprises et des acteurs locaux. Il y a ainsi une mise en communication de l'économie sociale et des autres composantes de l'économie mixte, ce qui permet d'envisager aussi bien la question de son intervention, de ses financements, que celle des carrières diversifiées ouvertes aux travailleurs, ceux-ci étant susceptibles de passer au cours du temps, d'un poste classique en entreprise privée, à une position de bénévole, avant d'intégrer comme salarié une organisation sans but lucratif. La mise en communication sécurisée de ces différentes activités devient en même temps un défi organisationnel et une opportunité démultipliant les choix collectifs en matière d'emploi et d'activité.

L'interrogation sur le contrôle des trajectoires et des modes de vie se dédouble ici. En effet, nous retrouvons d'abord, simplement transposée de la sphère de la production à celle du

\footnotetext{
${ }^{3} \mathrm{D}$ 'autres transitions sont évidemment possibles, celles qui relient par exemple chômage et activités domestiques, ou chômage et formation.
} 
marché du facteur travail, l'exigence de variété systémique qui fonde l'économie mixte. Mais d'autre part l'intervention des pouvoirs publics et des accords négociés organisant les transitions ne peut être efficace que dans le contexte d'un marché du travail maîtrisé, où les entreprises privées sont mises sous pression en quelque sorte de manière à ne pas pouvoir restreindre leur offre à des postes faiblement attractifs, ni trier indûment les candidats trop nombreux. On connaît l'aphorisme keynésien selon lequel la meilleure situation du marché du travail pour les travailleurs est le boom permanent, à la frontière du plein - emploi. La limite de cette situation se trouve évidemment dans les tensions inflationnistes que génère le plein emploi traditionnel, certains secteurs étant conduits à hausser leurs salaires pour attirer suffisamment de candidats. Les MTT retrouvent et reformulent cette intuition. Ils consistent à générer un ensemble de places suffisant pour permettre aux travailleurs d'exercer leur choix. Mais comme ils élargissent l'éventail des places disponibles en incluant notamment la formation et de multiples combinaisons entre emploi et autres activités, ils permettent de reculer l'échéance des tensions inflationnistes (l'arrivée de travailleurs mieux formés payés à un même salaire correspondant à une baisse du coût du travail, et les entreprises trouvant plus facilement les employés qu'elles recherchent).

Cette perspective rapidement brossée a suscité de nombreux débats quant à ses débouchés opérationnels selon la variété des situations nationales et locales, mais aussi quant à sa faisabilité générale. Très vite, les insuffisances de la définition fournie plus haut des «transitions » (définition négative et centrée sur l'acception traditionnelle et limitative de l'emploi typique) ont été mises en évidence par les chercheurs se réclamant des MTT. Ce processus de critique interne a rencontré certaines des critiques externes les plus importantes, celles qui doutaient de la capacité des MTT à infléchir significativement la dynamique excluante et segmentée des marchés du travail existants. En effet, on a vu que de très nombreuses «transitions » se passent au sein des firmes elles-mêmes. Elles incluent les passages temps complet - temps partiel, et retour au temps complet, la polyvalence et les modifications d'affectations en interne, la variabilité des horaires et des primes... Alors des instruments spécifiques permettent de les gérer selon les mêmes principes de négociation : les comptes - épargne - temps, les comptes - épargne - salariale..., etc. A chaque fois le principe est celui d'une restauration du contrôle des personnes en cas de fluctuations fortes et imprévues. Mais au-delà de ces instruments, c'est l'emprise des travailleurs sur leur entreprise qui est en question, soit le pouvoir des «stakeholders» au regard de celui des « shareholders ». La logique des MTT rencontre ici celle de la réforme de l'entreprise visant à faire reculer le pouvoir des actionnaires. C'est seulement à cette condition que les tendances à l'exclusion et à la segmentation pourront être combattues. Aux deux niveaux de contrôle envisagés plus haut, il faut adjoindre un troisième. Non seulement il est nécessaire de garantir la variété systémique et de mettre sous pression permanente le marché du travail, mais encore il convient de rééquilibrer les pouvoirs au sein des firmes privées, notamment mais pas exclusivement en ce qui concerne l'affectation et la rémunération des travailleurs.

L'ensemble de ces développements peut donc se résumer par la double exigence d' «équiper les gens pour le marché » et d' «équiper le marché pour les gens ». D'un côté on trouve les actions de formation et d'information, les actions visant à garantir à tous un accès aux emplois et donc à combattre les discriminations. Mais cette logique d'adaptation au marché du travail et d'ouverture de celui-ci est débordée par une seconde, qui s'inscrit en complément nécessaire. «Equiper le marché pour les gens », cela veut dire en contrôler le niveau d'activité, mais aussi créer les mécanismes collectifs par lesquels l'évaluation des personnes et leurs trajectoires débouchent sur des transitions socialement acceptables voire souhaitées. Ce ré-encastrement du marché du travail est nécessairement un processus ouvert, que ce soit 
sur les déterminants et politiques macro - économiques, ou sur l'organisation du secteur non marchand et domestique. Il convient désormais d'examiner celui-ci.

II.3. La construction sociale des «biens publics » et la redéfinition du rôle de l'Etat.

Les transformations récentes du rôle de l'Etat - Nation et notamment l'interdépendance accrue des politiques étatiques, ont ouvert un champ d'incertitudes et de débats sur les fondements et les limites de ces interventions. Ne sommes-nous pas très loin de l'émergence d'acteurs puissants et relativement autonomes qu'observait Polanyi ? De manière paradoxale, c'est à partir du cœur même de la pensée économique la plus traditionnelle que l'interrogation a pu prendre forme et rebondir. En effet, au fondement de la défaillance du marché se trouve le concept de bien public. Depuis les travaux classiques de Samuelson (Samuelson 1954), celui-ce se définit par deux traits négatifs, posant deux barrières au fonctionnement normal des mécanismes marchands. D'une part, un bien public est «non rival », c'est-à-dire que si un consommateur consomme de ce bien, il en reste autant pour d'autres consommateurs. D'autre part il est «non excluable », entendons par là qu'il est impossible (ou très coûteux) d'en interdire la consommation à un consommateur potentiel. Une telle définition est souvent illustrée par l'exemple canonique du phare, dont un marin peut profiter sans empêcher d'autres marins de le faire, et dont il est très difficile de faire payer l'usage par ses utilisateurs effectifs. Le processus usuel par lequel des entrepreneurs privés se chargent de produire puis de vendre est donc entravé, il s'agit d'un besoin collectif dont la satisfaction doit faire appel à une initiative collective.

L'approfondissement de ce concept a pris de multiples voies (Touffut (dir) 2006). La première a consisté à remarquer qu'il n'existe pas d'exemple totalement pur de bien public. Le phare, tout comme la défense nationale, peut en fait résulter d'initiatives privées marchandes. Des utilisateurs potentiels, par exemple les propriétaires de bateaux d'un port donné, ou des groupes soucieux de se protéger contre des menaces d'attentats, peuvent se regrouper spontanément et rémunérer un entrepreneur privé qui pourra construire un phare ou constituer une milice privée. Mais cette voie, qui affaiblit implicitement le concept en en faisant un cas - limite, et semble restaurer le rôle des initiatives privées marchandes, n'est que l'envers d'une autre qui fait de la non - rivalité et la non - excluabilité deux construits sociaux et politiques. L'exemple de la route à péage permet aisément de saisir ce point. La route semble un exemple typique de bien public, même si elle peut être sujette à congestion. Il est toutefois possible, dans certaines configurations géographiques, de construire des routes dont les points d'accès sont contrôlés et payants. C'est le cas de nombreuses autoroutes de longue distance. On observe toutefois qu'elles peuvent être gratuites, notamment dans les zones de forte densité urbaine et lorsque les accès se multiplient. Mais le choix entre gratuité et péage, qui dépend de ces aménagements, est en définitive un choix politique. On peut ainsi choisir d'organiser l'excluabilité ou la non - excluabilité. Cette seconde voie cesse d'être un constat défensif lorsque l'on réfléchit sur le champ qu'ouvre le constat de la construction sociale et politique de la rivalité et de l'excluabilité. En effet, en prenant appui sur l'encastrement - étayage discuté dans notre première section, on voit immédiatement que la définition d'un bien public ne peut se faire dans un vide social. La variabilité de la démarcation entre bien public et bien privé n'est donc pas tant le signe d'un flou que celui d'une série d'expériences et de projets. Elle suppose, pour certains biens susceptibles d'être concernés, et de manière variable dans le temps et dans l'espace, l'identification de priorités et l'obtention de compromis spécifiques. C'est ainsi que la dégradation de la qualité de l'air 
(bien public) peut conduire à une gestion purement privée (des bornes payantes permettant d'inhaler de l'air pur ou de l'oxygène) ou une réaction publique (des décisions collectives et contraignantes visant à restaurer la qualité de l'air). On conçoit bien du reste que les seuils de tolérance et de déclenchement des décisions privées et publiques soient variables selon les lieux, les groupes sociaux, etc.

Les constats et les analyses vues plus haut sur le rôle de l'Etat dans la nouvelle économie mixte sont ici repris au travers du prisme de la «nouvelle finance publique ». Elle apparaît sur un constat de décès : la fin de l'Etat «Westphalien » (Kaul et Conceiçâo 2006), celui qui précisément constituait l'horizon des observations de Polanyi. Selon ces deux auteurs, l'Etat «Westphalien» comme idéal - type superposait un espace géographique délimité, une population, une langue, une communauté de destins, une identité nationale et une unité décisionnelle, productrice et protectrice. L'argument économique classique se concentre sur la production de biens publics tels que la protection contre une agression militaire, l'ordre public, mais aussi bien sûr la justice, l'éducation de base, la santé publique ou la lutte contre la pauvreté, et qui tous ne sont pas produits, ou pas en quantité / qualité suffisantes, par les acteurs privés engagés dans les interactions de marché. Le point de départ est ainsi, comme on l'a va, la réponse à la défaillance des marchés par une série de prises de responsabilités, d'initiatives et d'exclusivités nationales et publiques. Nous retrouvons alors le circuit des défaillances discuté ci-dessus.

Cette première étape a d'ores et déjà conduit à un Etat régulateur, incitatif, facilitateur et partenarial, mixant un mélange de coopération et de concurrence. L'idée principale qui justifie l'apparition plus récente d'une «nouvelle finance publique » et la relecture du concept de bien public est au-delà. Les changements récents impliquent l'importance croissante des interdépendances internationales, mais aussi la reconnaissance et la production de biens publics «mondiaux». Soucieux d'attirer les capitaux étrangers, l'Etat nation est conduit à moduler ses politiques de taxation et à se soucier de sa réputation comme débiteur; la lutte contre le terrorisme transnational, la mise au point de réseaux internationaux de transports, la lutte contre les pandémies, la recherche d'une stabilité financière, la promotion des droits de l'homme sont autant de domaines qui n'impliquent pas simplement une coopération avec d'autres Etats mais bien avec d'autres acteurs privés, et la prise en compte de ces interactions n'est plus compatible avec la représentation traditionnelle de l'Etat centré sur son territoire et souverain dans ses décisions internes. L'Etat «Westphalien » est ainsi en train de céder la place à un Etat «intermédiaire » (op. cit. p. 73), qui doit en permanence combiner des préférences nationales et externes, corriger des marchés et se faire corriger par d'autres acteurs, notamment issus des marchés mondiaux. La souveraineté des acteurs de l'Etat nation, en charge de l'élaboration des politiques nationale, n'est plus exclusive mais interactive.

Ces mutations redéfinissent, complexifient et parfois élargissent le rôle de l'Etat - nation. Tout d'abord elles ne réduisent pas le champ de l'intérêt collectif mais au contraire l'étendent. Notamment l'arrivée de biens publics mondiaux et «régionaux », tels que ceux que nous avons évoqués ci-dessus élargit le jeu des interdépendances et appelle des mobilisations et coopérations nouvelles. A titre d'exemple, le fonctionnement harmonieux des marchés euxmêmes devient un bien public, avec par exemple la stabilité de la finance mondiale. Mais alors dans de nombreux cas l'Etat - nation apparaît, à l'échelle internationale, comme un acteur privé intéressé. Le cas difficile des coopérations dans le domaine du commerce international illustre bien la logique de l'intérêt égoïste qui prévaut la plupart du temps dans ce champ où les accords dépendent de l'implication volontaire de chaque nation. Lorsqu'il s'agit de l'effet de serre ou de la lutte contre une pandémie, l'Etat - nation prend une place 
centrale dans un processus impliquant les organisations internationales sans but lucratif et les instances publiques supranationales. Se constituent alors ce que Kaul et Conceiçao appellent des «composants d'action collective », à la géométrie et aux moyens différents dans chaque cas, visant à instaurer une coopération public - privé efficace face au problème posé. En définitive, l'Etat - nation perd le supposé monopole de la production des biens publics pour devenir le lieu central d'identification de l'intérêt collectif et l'agence de coordination évaluation des actions visant à y conduire. Cherchant à concilier les intérêts privés avec l'intérêt collectif, l'Etat peut ainsi avoir recours à une vaste gamme d'outils, tels que le partenariat, la régie, les appels d'offre... dont l'expérimentation s'affine au cours du temps.

II.4. Socialisme associationniste et socialisme libéral.

Karl Polanyi était socialiste. Dans La Grande Transformation, il rend hommage à Marx et à Robert Owen. Dans ses ouvrages et tout au long de sa vie, il a abordé la question du socialisme par divers voies : comme membre du mouvement des étudiants en Hongrie au début du $20^{\text {ème }}$ siècle, en tant qu'éducateur dans le mouvement syndical, en participant à des débats théoriques en économie sur la faisabilité du socialisme dans les années 20, où il est entré en combat intellectuel contre Ludwig Von Mises, et enfin en participant à des débats sur l'éducation socialiste en Angleterre.

Dans son débat avec Von Mises, il critiquait le modèle de la planification centrale, pour favoriser un socialisme démocratique, ou, dans ses mots, un «socialisme fonctionnel ». Il mettait en définitive l'accent sur la démocratie. Dans notre vocabulaire contemporain, il se focalisait plutôt sur la question de la gouvernance. Le débat initial avec Von Mises était, en un sens, plus facile. Il fallait seulement construire une argumentation pour contester la théorie de Von Mises sur l'impossibilité d'avoir un système des prix dans un système socialiste dépourvu de marchés libres. Polanyi a consacré 10 ans à l'élaboration d'un modèle théorique confirmant la capacité d'une économie socialiste d'arriver à produire et utiliser des prix. Dans l'histoire de la pensée économique, cette contribution reste comme un apport important dans l'étude des modèles comparatifs en économie. La position prise par Polanyi était hybride, parce qu'il était influencé plutôt par une conceptualisation basée sur l'individu (Bauer) que par l'approche de l'école historique (Schmoller).

Cette position et cette approche ont eu et ont encore des répercussions importantes dans les débats sur la démocratisation de l'économie. En fait, Polanyi a essayé de construire un modèle de "démocratie fonctionnelle », une démocratie associative, si on veut utiliser des concepts plus contemporains, qui, pour Polanyi, était à la base d'un socialisme démocratique. Pour lui, la question : comment construire le socialisme ? était plutôt une question organisationnelle. Pour y arriver et pour assurer son fonctionnement démocratique, il proposait la création d'associations de producteurs et de consommateurs en insistant sur le fait que nous sommes tous à la fois producteurs et consommateurs, et que nous devons nous organiser (créer des associations) afin d'assurer la meilleure représentation de nos doubles fonctions.

Polanyi était influencé par ses lectures de Robert Owen et G.D.H. Cole sur le «guild socialism ». Mais il était aussi très influencé, comme on l'a vu, par le socialisme municipal de 
la ville de Vienne ou il a résidé, jusqu'à son démantèlement en 1934. Le modèle du socialisme adopté à Vienne était participatif, inclusif et démocratique. L'accent était mis aussi sur l'éducation, sur la formation, sur la construction d'une connaissance reflétant la réalité de la vie des citoyens. Polanyi a beaucoup écrit sur l'importance de la construction d'une telle connaissance pour mobiliser l'action des individus et les collectivités. Il rejoignait en cela les sociaux-démocrates viennois de l'époque, pour qui la réforme scolaire et l'objectif de «socialiser» les citoyens par une «politique de la pédagogie» étaient fondamentaux. Selon Polanyi, la base de la démocratie associative existait dans les mouvements syndical et associatif, qui fournissaient des lieux de débats démocratiques ainsi que des lieux de collecte d'information/données, etc., nécessaire pour construire son modèle de «socialisme fonctionnel ».

Il est ainsi possible de revenir au modèle de démocratie fonctionnelle qui se trouve à la base même du socialisme («living essence of socialism») pour Polanyi. On a déjà introduit sa vision d'un socialisme associationiste. Pour cela, il estimait nécessaire de créer des organisations représentatives et fonctionnelles comme un congrès des producteurs (« kosten gruppen ») et une «Kommune », qui représente les citoyens en tant que consommateurs et en tant que membres d'une collectivité. Dans notre langage contemporain, le modèle qu'il proposait était ainsi d'orientation «bottom-up » au sens où les associations étaient les lieux décisionnels; le «haut» était plutôt une structure administrative qui mettait en œuvre les décisions prises par le «bas ». Et il insistait sur la capacité des syndicats, les associations industrielles, les coopératives et les municipalités socialistes à construire une telle économie socialiste.

De tels développements s'inscrivent dans une tradition ancienne, aujourd'hui vivante mais éclatée, celle du «socialisme libéral », que l'on peut distinguer aussi bien de la social démocratie que du «social - libéralisme» (Audier 2006). Trouvant ses premières formulations dans les œuvres de J.S. Mill et de L. T. Hobhouse pour l'Angleterre, de P. Leroux, A. Naquet, puis J. Jaurès et C. Gide pour la France, le socialisme libéral s'est ensuite épanoui en Italie avec les travaux de C. Rosselli et F.S. Merlino durant l'entre deux guerres, puis de G. Calogero. Issus de très nombreux pays développés, ses représentants récents vont de P. Calamandrei à C. Lefort en passant par J. Habermas, N. Bobbio et M. Walzer, et dialoguent avec A. Sen pour les enjeux des pays en voie de développement. Polanyi est ainsi un jalon dans la recherche toujours actuelle d'une combinaison intime entre socialisme et démocratieEn effet, à l'époque de Polanyi, les choix étaient assez tranchés entre un système du marché «auto - régulateur » et une planification centralisée. En ne proposant ni l'un ni l'autre, et en cherchant un modèle économique qui respectait avant tout la démocratie, Karl Polanyi n'entendait pas tempérer la radicalité de son projet politique, qui visait à dépasser le capitalisme, et non pas simplement admettre son développement comme inévitable en proposant d'en contre - balancer les excès par quelques interventions étatiques.

Le modèle proposé par Polanyi était de toute évidence incomplet. Mais il demeure aujourd'hui encore pertinent et suggestif dans le contexte des expérimentations actuelles de démocratisation économique, et les divers processus mis en œuvre pour y arriver. Il renforce notre capacité à conceptualiser le rôle de la société civile comme «agent », comme acteur clé dans la création des stratégies visant à démocratiser l'économie d'aujourd'hui. Son accent mis sur l'efficacité des associations, leur capacité à se mettre en réseaux, à dialoguer entre elles et à fournir à l'appareil de l'Etat (toujours essentiel) l'information nécessaire pour mieux mener 
des politiques publiques, est en résonance avec de nombreuses initiatives et théorisations actuellement en cours.

\section{Conclusion}

L'ambition de cet article était de mettre en évidence quelques unes des dynamiques induites aujourd'hui par l'œuvre de K. Polanyi, en privilégiant le couple encastrement désencastrement. Par delà le simplisme apparent de ce couple, nous avons pu montrer qu'il convenait désormais de faire justice à la fois à sa complexité et à son potentiel dérangeant. Sa résurgence au cœur même des développements d'Esping - Andersen témoigne tout d'abord de l'existence à la fois relativement stable et problématique de déclinaisons sociétales, les sociétés de type «anglo-saxon » s'avérant plus proches du marché que d'autres sociétés du monde développé, et générant des cultures compatibles avec ce type de régulation. Mais cet « encastrement - étayage » ne fait que réduire des tensions sans les faire disparaître. Symétriquement, les efforts de «réencastrement » identifiés dans la trajectoire du XXe siècle par B. Lévesque se font sous le signe de la défaillance, y compris ceux qui visent à promouvoir l'économie sociale. Il ne suffit pas d'affirmer et de mettre en œuvre un projet global affectant l'ensemble des domaines de l'activité économique et des échanges, celui-ci doit être réflexif et évoluer à mesure que les conditions de l'action publique se transforment. C'est pourquoi ce sont les liens intimes entre changement et incohérence qui passent au premier plan, suggérant l'intérêt d'un retour aux analyses polanyiennes du changement institutionnel et à leur insistance sur le désordre et le conflit.

La seconde étape de notre analyse a repris la même dynamique conceptuelle en passant au niveau des stratégies actuellement explorées et porteuses de nouvelles transversalités. Elle est ainsi partie d'une expérience spatialement et politiquement circonscrite, celle du Québec engagé dans un processus de plus en plus intégré, fondé d'abord sur l'économie sociale en tant que création collective d'instruments de production, mais élargissant ses projets et affirmant un pouvoir de négociation plus vaste. Elle a ensuite abordé deux autres stratégies de ré-encastrement : tout d'abord, le contrôle des «transitions » sur le marché du travail et autour de lui. On se retrouve alors dans le champ balisé par Esping - Andersen, celui des liens entre marché du travail et protection sociale, pour y tracer un cheminement partiel, expansif et transversal lui aussi. La troisième stratégie se focalise sur la redéfinition du rôle de l'Etat et le renouveau de l'idée d'intérêt général à travers la construction sociale de biens publics : troisième forme de transversalité, bousculant les spécialisations antérieures et réaffirmant la nécessité d'un engagement public. Dans ces trois cas, la pédagogie polanyienne de l'incohérence, reconnue ou non, est repérable et active, et c'est pourquoi la réflexion peut à nouveau faire retour sur ses projets les plus généraux, en les réinscrivant dans une démarche de transmission et de renouvellement.

Une lecture isolée de certains passages de La grande transformation pourrait accréditer une interprétation de K. Polanyi en termes de manichéisme diabolisant le marché. Le sentiment d'urgence inspiré par les convulsions des années trente et quarante a sans aucun doute joué un rôle essentiel dans la tonalité de ces passages. Mais l'œuvre est au-delà. Si l'on a pu créditer Joseph Schumpeter d'avoir montré qu'opérait un processus de «destruction créatrice » au sein du capitalisme, avec le recul on peut créditer Karl Polanyi, son contemporain, d'un apport symétrique et tout aussi général, sur les «contradictions créatrices du processus de 
désencastrement ». Liant intimement intérêts matériels et représentations, cette pensée continue à féconder les interrogations majeures de notre temps. Un de leurs paradoxes principaux, parfaitement fidèle à Polanyi, est que la création ou l'aménagement de marchés convenablement «équipés », par exemple distribuant ou finançant les produits de l'économie sociale, ou encore aménageant des «transitions » sur le marché du travail, est devenue une des voies du réencastrement.

\section{Références}

AUDIER, S. (2007), Le socialisme libéral, La Découverte

BEN-NER A. (2006), «Comment répartir la production des biens ? », in Touffut J. - P. (dir) 2006, L'avancée des biens publics, Politique de l'intérêt général et mondialisation, Albin Michel, pp 55 - 86

BEN - NER A. et GUI B. (dir) 1991, « The non-profit sector in the mixed economy », Annals of Public and Cooperative Economics, vol. 62, oct. - déc.

BEN - NER A. et VAN HOOMISSEN T. (1991), "Nonprofit organizations in the mixed economy. A demand and supply analysis", Annals of Public and Social Economy, vol. 62, oct. - déc., pp 519 - 550.

BUGRA, A. et AGARTAN, K. (dir) (2007). Reading Karl Polanyi for the Twenty-first Century. Market Economy as a Political Project. Palgrave.

CAILlE A. et LAVILLE J.L. (2007), « Actualité de Karl Polanyi », Revue du MAUSS n 29 , premier semestre, pp $80-109$

EME, B. et LAVILLE, J-L. (dir) (1994) Cohésion sociale et emploi. Desclée de Brouwer EME, B. et LAVILLE, J-L (2006) «Economie solidaire 2 ». Dictionnaire de l'autre économie. Laville, J-L. et Cattani, A.D. (dir). Gallimard, pp.303-312. ESPING - ANDERSEN G. (1990), The Three Worlds of Welfare Capitalism, Polity Press GAZIER B. (1986), "Pauvreté, risque, société", Bulletin du MAUSS, n 19, septembre 1986, pp $99-114$

GAZIER B. (1993), «L'économie sociale dans l'économie mixte aujourd'hui », Revue des études coopératives, mutuellistes et associatives, $\mathrm{n}^{\circ}$ 44/45, pp 174 - 182

GAZIER B. (2005) Vers un nouveau modèle social, Flammarion, coll. « Champs » GAZIER B. (2006), « « Marchés transitionnels du travail » : emploi durable et économie sociale », in Paugam S. (dir) 2006, Repenser la solidarité au XXIe siècle, PUF, pp 491 - 511 GAZIER B. et LECHEVALIER A. (2006), "Stratégie Européenne de l'Emploi, régimes macroéconomiques et institutionnels, et Marchés Transitionnels du Travail", in A.T. Dang, J.L. Outin et H. Zajedela (dir), Travailler pour être intégré ? Mutations des relations entre emploi et protection sociale, CNRS Editions, pp 218 - 234

GAZIER B. et TOUFFUT J.P. (2006), «Introduction. Bien public, bien social », in Touffut J. - P. (dir) 2006, L'avancée des biens publics, Politique de l'intérêt général et mondialisation, Albin Michel, pp 9 - 22

GAZIER B. (2007), « Marchés transitionnels du travail et insertion par l'activité économique », Economie et Humanisme, n 381, juin, pp 34 - 37

GIDDENS A. (1999), The Third Way. The Renewal of Social Democracy, Cambridge, Polity Press

GRANOVETTER M. (1985), «Economic action and social structure: the problem of embeddedness », American Journal of Sociology, vol 91, n³, pp 481 - 510 
GRANOVETTER M. (2000), Le marché autrement, Desclée de Brouwer HARVEY, M., RAMLOGAN,R. et RANDLES, S. (dir) (2007). Karl Polanyi, New Perspectives on the Place of the Economy in Society. University of Manchester Press.

KAUL I. (2006), «Une analyse positive des biens publics », in Touffut Jean - Philippe (dir) 2006, L'avancée des biens publics, Politique de l'intérêt général et mondialisation, Albin Michel, pp 23 - 54

KAUL I., et CONCEICAO P. (dir) 2006, The New Public Finance, Responding to Global Challenges, Oxford U. Press 2006

HOLLINGSWORTH J.R., et BOYER R. (dir) 1997, Contemporary Capitalism. The Embeddedness of Institutions, Cambridge U. Press

LAVILLE, J-L. (1996) «Economie et solidarité : linéaments d'une problématique », in OCDE : Réconcilier l'économique et le social. L'économie plurielle. Paris, OCDE.

LE VELLY R. (2007), "Le problème du désencastrement”, Revue du MAUSS, n² 29, premier semestre, pp 181 - 196

LEVESQUE B. (2003), «Fonction de base et nouveau rôle des pouvoirs publics : vers un nouveau paradigme de l'Etat », Annals of Public and Cooperative Economics/Annales de l'Economie Publique, Sociale et Coopérative, vol. 74, n 4, December, pp 489 - 513

McCROBBIE, K. et POLANYI-LEVITT, K. (2006). Karl Polanyi in Vienna : The

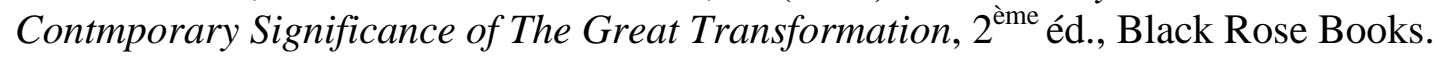
MAUCOURANT Jérôme, 2005, Avez-vous lu Polanyi ? La socio-économie de Karl Polanyi, La Dispute

MENDELL, M et POLANYI-LEVITT, K (1987) "Karl Polanyi. His Life and His Times", Studies in Political Economy. No.22, Spring 1987. pp. 7-39.

MENDELL M. (1990), « Karl Polanyi and Feasible Socialism », in Polanyi Levitt K. (dir), The Life and Work of Karl Polanyi, Montréal, Black Rose Books, pp 66 - 77

MENDELL M. (1994), « Karl Polanyi and Socialist Education », in McRobbie K. (dir) 1994, Humanity, Society and Commitment, Montréal, Black Rose Books, pp 25 - 44.

MENDELL, M. (2002), "The Social Economy in Québec. Discourse and Strategies" in MacDonald E. et Bakan A. (dir) Critical Political Studies: Debates and Dialogues for the Left. Montréal: McGill-Queen's Press, pp. 468-502.

MENDELL, M (2007a), "Karl Polanyi and the Instituted Process of Economic Democratization" in M. Harvey, R. Ramlogan and S. Randles (dir), Polanyian Perspectives on Embedded Economic Processes, Development and Transformation. Manchester : Manchester University Press. pp. 78-92.

MENDELL M. (2007b), "The Social Economy: Diverse Approaches and Practices in Europe and Canada" in A. Noya and E. Clarence, (dir) The Social Economy. Building Inclusive Economies, France: OECD Publications, 2007. pp. 155-187. (Avec J-L. Laville et B.

Lévesque].

MENDELL M. (2007c), "Karl Polanyi et le processus institué de démocratisation politique », Revue du MAUSS, $n^{\circ} 29$, premier semestre, article électronique

MENDELL, M. (2007d), «La co-producción de politicas sociales en Québec : el caso de la economia social », in Vuotto M. (dir). La co-construcción de políticas en el campo de la economía social. Prometeo, pp. 19-27.

POLANYI K (1977), The Livelihood of Man, Academic Press

POLANYI K. (1983) La grande transformation. Aux origines politiques et économiques de notre temps, Gallimard (Edition originale en langue anglaise 1944)

POLANYI K. (2007), « Le sophisme économiciste », Revue du MAUSS n 29, pp 63 - 79

(traduction revue d'extraits de The Livelihood of Man)

POLANYI K. (2008), Essais, Editions du Seuil

Politics and Society (2003), Special Issue "The Legacy of Karl Polanyi". Vol.31, No.2. 
ROUSTANG, G., LAVILLE, J-L., EME, B., MOTHE, D. et PERRET, B. (1997). Vers un nouveau contrat social. Desclée de Brouwer.

SAMUELSON P. (1954), «The Pure Theory of Public Expenditure », Review of Economics and Statistics, 36 (4), pp 387 - 389

SCHMID G., et GAZIER B. (2002), (dir) The Dynamics of Full Employment. Social Integration by Transitional Labour Markets, Edward Elgar, 443 p.

SCHMID G. (2006a), "Social Risk Management through Transitional Labour Markets", Socio-Economic Review 4 (1), 1-33

SOBEL R. (dir) (2007), Penser la marchandisation du monde avec Karl Polanyi, L'Harmattan / Cahiers Lillois d'Economie et de Sociologie SULLIVAN, H. et SKELCHER, C. (2002), Working Across Boundaries. Collaboration in Public Services. Palgrave Macmillan. 271p.

TOUFFUT J. - P. (dir) (2006), L'avancée des biens publics, Politique de l'intérêt général et mondialisation, Albin Michel 